Published in final edited form as:

J Math Biol. 2008 August ; 57(2): 161-181.

\title{
Maintaining phase of the crustacean tri-phasic pyloric rhythm
}

\author{
Christina Mouser ${ }^{*}$, Farzan Nadim ${ }^{\dagger}$, and Amitabha Bose ${ }^{\ddagger}$ \\ ${ }^{*}$ Department of Mathematical Sciences, Medgar Evers College of CUNY, Brooklyn, NY 11225: \\ cmouser@mec.cuny.edu \\ $\dagger$ Department of Mathematical Sciences, New Jersey Institute of Technology, Newark, NJ 07102: \\ farzan@njit.edu \\ \$ Department of Mathematical Sciences, New Jersey Institute of Technology, Newark, NJ 07102: \\ bose@njit.edu
}

\begin{abstract}
We construct and analyze a model network of the pyloric rhythm of the crustacean stomatogastric ganglion consisting of an oscillator neuron that inhibits two reciprocally inhibitory follower neurons. We derive analytic expressions that determine the phase of firing of the follower neurons with respect to the oscillator. An important aspect of the model is the inclusion of synapses that exhibit shortterm synaptic depression. We show that these type of synapses allow there to be a complicated relationship between the intrinsic properties of the neurons and the synapses between them in determining phase relationships. Our analysis reveals the circumstances and ranges of cycle periods under which these properties work in concert with or independently from one another. In particular, we show that phase maintenance over a range of oscillator periods can be enhanced through the interplay of the two follower neurons if the synapses between these neurons are depressing. Since our model represents the core of the oscillatory pyloric network, the results of our analysis can be compared to experimental data and used to make predictions about the biological network.
\end{abstract}

\section{Keywords}

oscillator; phase plane; central pattern generator; synaptic depression

\section{Introduction}

Neuronal networks responsible for the generation of rhythmic motor activity often operate over a broad range of frequencies [18]. In many such oscillatory central pattern generating (CPG) networks the relative activity phase between groups of neurons remains fixed despite large variations in network frequency $[6,10,11,13,14,25]$. Hence, in face of changes in network frequency, the time delay between the active states of these neuron groups must be adjusted proportionally with the network cycle period. Yet, the biological mechanisms underlying such adjustments are largely unknown. Previous modeling studies have addressed the question of phase determination in neuronal networks. There is a large literature on weakly coupled oscillators (see [8] for example) in which the phase relationship between neurons is obtained through methods of averaging. In studies of the lamprey CPG, the phase between neurons is explicitly constrained within the model [5], allowing the authors to investigate what mechanisms are consistent with the constraint. Work on understanding the inter-segmental lag in the crayfish CPG has focused on the interaction between weak and strong coupling [15, 30].

In previous work, we have suggested that short-term synaptic depression helps promote phase maintenance in feed-forward networks. We showed that depression in the feed-forward 
inhibitory synapse from an oscillator to a follower neuron allows the phase to be fairly constant over a large range of cycle periods [17] and that intrinsic ionic currents such as the transient potassium A current can act synergistically with synaptic depression to extend the range of phase maintenance [4]. In the current study, we focus on a CPG neuronal network to examine phase maintenance of follower neurons in a three-cell network in which a pacemaker neuron imposes the network oscillations on two follower neurons coupled with reciprocally inhibitory synapses (inset of Fig. 1). The questions of interest center on the synaptic and intrinsic mechanisms that control the activity phase of the follower neurons and their dependence on cycle frequency. This network is modeled after the pyloric network of the crustacean stomatogastric ganglion which consists of an oscillatory pacemaker group of neurons $A B$ and $P D$ and four sets of follower neurons [20]. The pacemaker $A B$ and $P D$ neurons are always coactive, due to strong electrical coupling, and inhibit all follower neurons which, in turn, become active in two distinct (but sometimes overlapping) intervals in each cycle, thus producing a triphasic rhythm (Fig. 1A). Previous experimental studies have shown that the activity phases of all neurons in this network are relatively well maintained, despite large variations $(0.5-2 \mathrm{~Hz})$ in cycle frequency $[13,14]$.

In this study, we use the $A B$ neuron as the representative of the pacemaker group and the follower neurons $L P$ and $P Y$ as representatives of the follower neurons active in two distinct phases. The other pacemaker neuron type $P D$ two follower neuron types $I C$ and $V D$, which are co-active with $L P$ and $P Y$, respectively, are not included in our model. Thus, in our model, $A B$ sends feed-forward inhibition to $L P$ and $P Y$ while the latter two neurons reciprocally inhibit one another.

We use phase-plane analysis to derive a set of equations that can be numerically solved at any value of the period to determine the phase relationship between $A B$ and the follower neurons. As we vary the cycle period of $A B$, we investigate how the activity phases of $L P$ and $P Y$ changes. The analysis allows us to determine the role of each of the neurons and the synapses between them in setting their activity phases. In particular, we show that when all synapses exhibit shortterm depression, there are multiple control mechanisms for setting the phases. These control mechanisms may work in concert or be completely independent from one another. We show that as the cycle period changes, the control of the network phase relationships shifts between different sets of parameters associated with the neurons and their synapses. Our results, therefore, indicate that synaptic depression within the pyloric network provides a natural mechanism to help the network maintain the observed phase relationships among its different neurons despite large changes in the cycle period.

The mathematical techniques that we employ are motivated by geometric singular perturbation theory in which a difference in time scales is exploited to reduce a high-dimensional model to low-dimensional sub-systems that are more amenable to analysis [21]. We utilize this idea to project the dynamics of the neurons in their silent state onto a two- or three-dimensional phase space. Analyzing the ensuing dynamics in these phase spaces then makes it possible to analytically determine how long each neuron spends in its silent state, thereby allowing us to calculate the phase of each neuron. In parallel, we numerically solve the full set of model equations to see how these numerical solutions compare with those obtained from the analytic reduction method.

The paper is organized as follows. In section 2, we derive the model set of equations and describe the dynamics of synaptic depression. Section 3 contains results with section 3.1 containing the derivation of the firing times and subsequent subsections discussing the role of each of the synapses within the network. Section 4 contains a Discussion in which we address some of the modeling assumptions and implications for the biological network. 


\section{Model}

The network we are studying consists of three neurons, $A B, L P$, and $P Y$. A schematic of the network architecture is shown in Fig. 1 together with biological and simulation voltage traces of these neurons. $A B$ is the pacemaker neuron of the pyloric network. It oscillates at frequencies that lie between 0.5 and $2.0 \mathrm{~Hz}$ [24]. The activity of the pacemaker $A B$ neuron is taken for simplicity to be a square-wave. In particular, we denote the time that $A B$ is active by $T_{A B}$ and the time it is inactive by $T_{\text {inactive }}$. Therefore the period of $A B$ satisfies $P=T_{A B}+T_{\text {inactive }} . L P$ and $P Y$ are the follower neurons of the pyloric network. We are mostly interested in the burst envelope of these neurons so we use Morris-Lecar type equations to model their activity [22]. The equations to describe the activity of $L P$ and $P Y$ without synaptic connectivity are:

$\frac{d v_{x}}{d t}=I_{x}-g_{\text {leak }}\left[v_{x}-E_{\text {leak }}\right]-g_{C a} m_{\infty}\left(v_{x}\right)\left[v_{x}-E_{C a}\right]-g_{K} w_{x}\left[v_{x}-E_{K}\right]$

$$
\frac{d w_{x}}{d t}=\frac{w_{\infty}\left(v_{x}\right)-w_{x}}{\tau_{w, x}\left(v_{x}\right)}
$$

where $x$ is $P Y$ or $L P . v_{x}$ is the voltage of neuron $x, I_{x}$ in an applied current, $g_{l e a k, x}$ is the conductance of the leak current, $E_{l e a k, x}$ is the reversal potential of the leak current, and $w_{x}$ is the recovery variable of $K^{+}$. The functions $m_{\infty}, w_{\infty}$, and $\tau_{\infty}$ are sigmoidal functions of voltage and are given in the Appendix. Recent experiments by Rabbah et al [27] showed that the delay in firing of $P Y$ relative to firing of $L P$ is due to intrinsic properties of the neurons. Thus, in the silent state, we choose $\tau_{w, L P}\left(v_{L P}\right)<\tau_{w, P Y}\left(v_{P Y}\right)$ so that $P Y$ has a a slower intrinsic decay rate than $L P$.

We denote the right-hand side of (1) by $f\left(v_{x}, w_{x}\right)$. The $v_{x}$-and $w_{x}$-nullclines associated with (1) and (2) are the set of points $\left\{\left(v_{x}, w_{x}\right): f\left(v_{x}, w_{x}=0\right\}\right.$ and $\left\{\left(v_{x}, w_{x}\right): w_{x}=w_{\infty}\left(v_{x}\right)\right\}$. The former is cubic shaped, the latter is sigmoidal. We assume that for both $L P$ and $P Y$, these nullclines intersect at a stable fixed point along the right branch of the cubic nullcline. Thus in the absence of input, both $L P$ and $P Y$ tend to a high-voltage fixed point.

$L P$ and $P Y$ receive inhibitory synaptic inputs from $A B$ and also from one another. The synapses are modeled to be depressing meaning that the strength of the synapse weakens as the frequency of the pre-synaptic neuron increases. The equations to model the activity of $L P$ and $P Y$ are:

$$
\begin{gathered}
\frac{d v_{L P}}{d t}=f\left(v_{L P}, w_{L P}\right)-\bar{g}_{A B} s_{A B}\left[v_{L P}-E_{i n h}\right]-\bar{g}_{P Y} s_{P Y}\left[v_{L P}-E_{i n h}\right] \\
\frac{d w_{L P}}{d t}=\frac{w_{\infty}\left(v_{L P}\right)-w_{L P}}{\tau_{w, L P}\left(v_{L P}\right)} \\
\frac{d v_{P Y}}{d t}=f\left(v_{P Y}, w_{P Y}\right)-\bar{g}_{A B} s_{A B}\left[v_{P Y}-E_{i n h}\right]-\bar{g}_{L P} s_{L P}\left[v_{P Y}-E_{i n h}\right] \\
\frac{d w_{P Y}}{d t}=\frac{w_{\infty}\left(v_{P Y}\right)-w_{P Y}}{\tau_{w, P Y}\left(v_{P Y}\right)}
\end{gathered}
$$

In the above equations, $\bar{g}_{A B}, \bar{g}_{P Y}$ and $\bar{g}_{L P}$ are the maximal conductances of the $A B, P Y$ and $L P$ synapses, respectively, while $s_{A B}, s_{P Y}$ and $s_{L P}$ are the associated gating variables. $E_{i n h}$ is the reversal potential of the synapses that is chosen so that all synapses are inhibitory.

Our model for the synapses is similar to that in [17] where each synapse has a gating variable $s_{x}$ and another variable $d_{x}$ that measures the extent of synaptic depression of the synapse. Both 
variables follow piecewise continuous linear kinetics. They are coupled at discrete moments of time whenever a pre-synaptic cell becomes active. When this occurs, $s_{x}$ is set equal to the current value of $d_{x}$; see Fig. 2. At all other times the equations for the each of the synaptic variables are:

$$
\begin{aligned}
& \frac{d d_{A B}}{d t}= \begin{cases}\left(\widehat{d}_{A B}\left(T_{\text {inactive }}\right)-d_{A B}\right) / \tau_{\alpha} & v_{A B} \leq v_{T} \\
-d_{A B} / \tau_{\beta} & v_{A B}>v_{T}\end{cases} \\
& \frac{d s_{A B}}{d t}= \begin{cases}-s_{A B} / \tau_{K} & v_{A B} \leq v_{T} \\
-s_{A B} / \tau_{\zeta} & v_{A B}>v_{T}\end{cases} \\
& \frac{d d_{P Y}}{d t}= \begin{cases}\left(\widehat{d}_{P Y}\left(P, T_{P Y}\right)-d_{P Y}\right) / \tau_{\alpha} & v_{P Y} \leq v_{T} \\
-d_{P Y} / \tau_{b} & v_{P Y}>v_{T}\end{cases} \\
& \frac{d s_{P Y}}{d t}= \begin{cases}-s_{P Y} / \tau_{1} & v_{P Y} \leq v_{T} \\
-s_{P Y} / \tau_{2} & v_{P Y}>v_{T}\end{cases} \\
& \frac{d d_{L P}}{d t}= \begin{cases}\left(\widehat{d}_{L P}\left(P, T_{L P}\right)-d_{L P}\right) / \tau_{C} & v_{L P} \leq v_{T} \\
-d_{L P} / \tau_{d} & v_{L P}>v_{T}\end{cases} \\
& \frac{d s_{L P}}{d t}= \begin{cases}-s_{L P} / \tau_{3} & v_{L P} \leq v_{T} \\
-s_{L P} / \tau_{4} & v_{L P}>v_{T}\end{cases}
\end{aligned}
$$

For example, when $A B$ enters its active state, we set $s_{A B}=d_{A B}$ and then let $d_{A B}$ and $s_{A B}$ evolve according to (7) and (8). $v_{T}$ is the activation threshold of the synapses. When $v_{A B}<v_{T}, d_{A B}$ increases to $\hat{d}_{A B}\left(T_{\text {inactive }}\right)$ (recovery from depression) with rate $1 / \tau_{\alpha}$ where $\hat{d}_{A B}\left(T_{\text {inactive }}\right)$ is an increasing function of $T_{\text {inactive }}$ given by

$$
\widehat{d}_{A B}\left(T_{\text {inactive }}\right)=\left(1+\tanh \left(\left(T_{A B}+T_{\text {inactive }}-P_{1}\right) / x_{1}\right) / 2 .\right.
$$

In this equation, $P_{1}$ determines the half-activation time of $\hat{d}_{A B}$ and $x_{1}$ determines the steepness of $\hat{d}_{A B}$. This relationship models the dependency of recovery from depression on the cycle period of activity and is used to provide a better approximation of the two time scales of recovery observed in the pyloric synapses [3]. Once $v_{A B}$ goes above threshold ( $A B$ becomes active), $s_{A B}$ is set to $d_{A B}$ and then decreases at a rate of $1 / \tau_{\zeta}$ where $\tau_{\zeta}$ is a large parameter. While $v_{A B}$ remains above threshold, $d_{A B}$ decreases towards 0 with time constant $1 / \tau_{\beta}$ representing the depression of the $A B$ synapse. Once $v_{A B}$ goes below threshold, $s_{A B}$ decreases to 0 with rate $1 /$ $\tau_{\kappa}$. As the inactive phase of $A B$ increases, the synapse has more time to recover from depression and thus $d_{A B}$ has more time to increase towards $\hat{d}_{A B}\left(T_{\text {inactive }}\right)$.

Similarly, we let

$$
\widehat{d}_{L P}\left(P, T_{L P}\right)=\left(1+\tanh \left(\left(P-T_{L P}-P_{2}\right) / x_{2}\right)\right) / 2,
$$

where $T_{L P}$ refers to the length of time $L P$ is active. Note that $T_{L P}$ is a priori unknown, but will be determined below. $\hat{d}_{P Y}\left(P ; T_{P Y}\right)$ can also be modeled using a sigmoid similar to $\hat{d}_{A B}$ where $T_{P Y}$ refers to the length of time that $P Y$ is active. For simplicity, however, we set $\hat{d}_{P Y}=1$.

In some cases that we will examine, the synapses from $A B$ to $L P$ and $P Y$ will be non-depressing. In these cases, the strength of the $A B$ synapses will be independent of period. Whenever $A B$ becomes active, we will set $s_{A B}=1$ instead of $d_{A B}$. 
The mathematical effect of inhibition is to lower the $v_{x}$-nullcline of the post-synaptic cell in the $\left(v_{x} ; w_{x}\right)$ phase space. Consider $L P$ for example. Before it receives inhibition from the pacemaker $A B, L P$ lies at a high-voltage fixed point. The inhibition from $A B$ turns on quickly and causes this fixed point to disappear, thereby allowing $L P$ to return to its silent state. Depending on the strength of the $A B$ inhibition (which due to depression is period dependent), the $v_{L P}$ - and $w_{L P}$-nullclines may intersect either on the middle or left branch of the cubic. In the former case, $L P$ will be able to leave the silent state due to its intrinsic properties, while in the latter $L P$ will remain in the silent state until the $A B$ inhibition decays; Fig. 3. The general effect of $P Y$ inhibition on $L P$ and of $A B$ and $L P$ inhibition on $P Y$ is similar.

We will not carry out a formal singular perturbation analysis in this paper. Instead, we will assume that the $w_{x}, s_{x}$ and $d_{x}$ variables evolve more slowly than the $v_{x}$ variables while the trajectory is away from the branches of a cubic nullcline. This can be achieved by taking the time constants associated with those variables to be large and will allow us to project the dynamics of the neurons while they are near the left branch of a cubic onto a lower dimensional phase space; see $[1,4]$ for related examples. Simulations of our model are done using the software package XPPAUT [9]. Numerical solutions of equations (25) and (26), below, were obtained using MATLAB. Parameter values are given in the Appendix.

\section{Results}

We describe the tri-phasic pyloric rhythm starting with the onset of $A B$ activity at $t=0$ as shown in Fig. 1. $A B$ remains active for time $T_{A B}$ during which time both $L P$ and $P Y$ are silent. At $t=$ $t_{1}, L P$ becomes active for a time length $T_{L P}$. During this time, it inhibits $P Y$. At $t=t_{2}, P Y$ becomes active and inhibits $L P$ enough to return $L P$ to the silent state. $P Y$ stays active for a time length $T_{P Y}$. After one period $P=T_{A B}+T_{\text {inactive }}, A B$ again becomes active, inhibiting $P Y$ and $L P$ and the cycle continues. We define $\varphi_{L P}=t_{1} / P$ and $\varphi_{P Y}=t_{2} / P$ as the phase at which $L P$ and $P Y$ fire with respect to the onset of $A B$ activity. The main goal of this paper is to determine these two quantities as a function of $P$, and to show what effect synaptic depression has on these two phases. Throughout this paper, we shall change $P$ by changing the time $T_{\text {inactive }}$ while keeping $T_{A B}$ fixed; see the Discussion for comments on how changing period in different ways affects the results.

To determine $\varphi_{L P}$ and $\varphi_{P Y}$, we must determine $t_{1}, t_{2}, T_{L P}$ and $T_{P Y}$. However the latter two quantities obey $T_{L P}=t_{2}-t_{1}$ and $T_{P Y}=P-t_{2}$. Thus we need only determine two equations for the remaining unknowns $t_{1}$ and $t_{2}$. To calculate these times we first must understand how $L P$ and $P Y$ evolve in their silent state, and what causes them to have a chance to jump to the active state. Consider first $L P$ in the case where only $A B$ inhibition is present. In the $v_{L P}-w_{L P}$ phase plane, inhibition lowers the $v_{L P}$-cubic nullcline. As $L P$ evolves in the silent state, the synapse from $A B$ decays, causing the cubic nullcline to rise slowly back toward its original location. $L P$ can jump to the active state when it reaches a local minimum of any of the slowly rising cubics. These local minima form a one-dimensional $L P$-jump curve in $\left(v_{L P}, w_{L P}\right)$ space; labeled j.c. in Fig. 4A.

Another way to visualize this is in the $\left(w_{L P}, s_{A B}\right)$ phase space; Fig. 4B. When projected into this phase space, the trajectory moves down and to the left. In addition to the $L P$-jump curve, there exists a curve of fixed points, labeled $f p . c$. . These points are not true fixed points of the system, but rather are points along which the $v_{L P}$ - and $w_{L P}$-nullclines intersect $\left(v_{L P}^{\prime}=w_{L P}^{\prime}=0\right)$, but where $s_{A B}^{\prime}<0$. A typical trajectory in this phase space will transition to the active state when it reaches the jump curve. Let us now add in the $P Y$ inhibition to $L P$. In this case, $L P$ must potentially wait for both the $A B$ and $P Y$ inhibitions to decay in order to jump to the active state. The local minima of the associated cubics now form a two-dimensional jump surface (j.s.) and the fixed points form a two-dimensional surface (fp.s.) as shown in the 
$\left(w_{L P}, s_{A B}, s_{P Y}\right)$ phase space in Fig. 4C. Similarly, there exists a two-dimensional $P Y$-jump surface resulting from the slow decay of the $A B$ and $L P$ synapses to $P Y$ and a two-dimensional surface of fixed points.

Let us assume that $A B$ becomes active at $t=0$ and that both $L P$ and $P Y$ are in their silent state. $L P$ is the first to reach its jump surface, primarily because of the assumption that when the neurons are below threshold, $\tau_{w, L P}\left(v_{L P}\right)<\tau_{w, P Y}\left(v_{P Y}\right)$. Assume that the jump surface is a plane and can be expressed by the linear relationship:

$$
\bar{g}_{A B} s_{A B}+\bar{g}_{P Y} s_{P Y}+M_{1} w_{L P}=g_{s y n}^{*} \text {. }
$$

Note that if $\bar{g}_{P Y}=0,(15)$ reduces to the equation for a one-dimensional linear jump curve as in [4]. The constants $M_{1}$ and $g_{s y n}^{*}$ are both positive and can be determined from the $\bar{g}_{P Y}=0$ case. The former is related to the slope of the $L P$-jump curve; the latter is related to the level of inhibitory synaptic input needed to make the $v_{L P}$-nullcline tangent to the $w_{L P}$-nullcline; see figure $4 \mathrm{~B}$. We now calculate the value at time $t_{1}$ of the three dynamic variables explicitly represented in (15). The first is the easiest. Since $A B$ is taken to be a periodic square wave function (with period $P=T_{A B}+T_{\text {inactive }}$ ), the value of the depression variable $d_{A B}$ will also be periodic. Using this fact, it is straightforward to find from (7) that the maximal value of $d_{A B}$ occurs at the onset of $A B$ activity and is given by

$$
d_{\text {max }, A B}(P)=\widehat{d}_{A B}\left(T_{\text {inactive }}\right) \frac{\left[1-e^{-T_{\text {inactive }} / \tau_{\alpha}}\right]}{1-e^{-T_{A B} / \tau_{\beta}} e^{-T_{\text {inactive }} / \tau_{\alpha}}} .
$$

Note that $d_{\max , A B}(P)$ is an increasing function of $T_{\text {inactive. }}$. At the time that $A B$ becomes active $s_{A B}$ is set equal to $d_{\max , A B}$ and then decays according to (8). Thus $s_{A B}\left(T_{A B}\right)=d_{\max , A B}(P)$

$e^{-T}{ }_{A B} / \tau_{\zeta}$. During the time that $A B$ is silent, $s_{A B}$ decays with time constant $\tau_{\kappa}$, so

$$
S_{A B}\left(t_{1}\right)=d_{m a x, A B}(P) e^{-T_{A B} / \tau_{\zeta}} e^{-\left(t_{1}-T_{A B}\right) / \tau_{K}} .
$$

Note that the case when $A B$ is not depressing can be considered by simply setting $d_{\text {max }, A B} \equiv$ 1. We next calculate $s_{P Y}\left(t_{1}\right)$. Since the tri-phasic rhythm is assumed to be periodic, we similarly find that

$$
d_{m a x, P Y}\left(P, T_{P Y}\right)=\frac{\left[1-e^{-\left(P-T_{P Y}\right) / \tau_{\alpha}}\right]}{1-e^{-T_{P Y} / \tau_{b}} e^{-\left(P-T_{P Y}\right) / \tau_{\alpha}}} .
$$

This maximum occurs at the onset of the $P Y$ burst. Note that $d_{\max , P Y}$ depends on $T_{P Y}$ where this term is to be determined by $t_{2}\left(T_{P Y}=P-t_{2}\right)$. Utilizing the activity of the previous cycle of a periodic solution, $t=0$ corresponds to the end of a $P Y$ burst. Therefore $s_{P Y}(0)=d_{\max , P Y}$ $e^{-T^{P Y} \tau_{2}}$ and at time $t_{1}$

$$
s_{P Y}\left(t_{1}\right)=d_{\max , P Y}\left(P, T_{P Y}\right) e^{-T_{P Y} / \tau_{2}} e^{-t_{1} / \tau_{1}}
$$

Finally, we determine $w_{L P}\left(t_{1}\right)$. When $P Y$ becomes active (during the previous cycle) assuming $\bar{g}_{P Y}>0, L P$ returns to the silent state with a value we denote $w_{L P} L P$ stays in the silent state for time $T_{P Y}+t_{1}=P-\left(t_{2}-t_{1}\right)$. Assuming that $w_{\infty}(L P) \approx 0$ near the left branches of the $v_{L P}$ nullcline and that $\tau_{w, L P}\left(v_{L P}\right)$ is a constant denoted by $\tau_{w, L P}$, we find

$$
w_{L P}\left(t_{1}\right)=\widehat{w}_{L P} e^{-\left(P-\left(t_{2}-t_{1}\right)\right) / \tau_{w, L P}} .
$$


We can now substitute equations (17)-(20) into (15). Note however that if $\bar{g}_{P Y}=0$, then $L P$ stays in the active state until $A B$ becomes active and (20) is replaced by $w_{L P}\left(t_{1}\right)=\hat{w}_{L P}$ $e^{-t^{1} / \tau^{w, L P}}$

To find a second equation relating the times $t_{1}$ and $t_{2}$, we use a linear approximation for the $P Y$-jump surface:

$$
\bar{g}_{A B} s_{A B}+\bar{g}_{L P} s_{L P}+M_{2} w_{P Y}=g_{s y n}^{*} .
$$

Similar to before,

$$
s_{A B}\left(t_{2}\right)=d_{\max , A B}(P) e^{-T_{A B} / \tau_{\zeta}} e^{-\left(t_{2}-T_{A B}\right) / \tau_{\kappa}} .
$$

To calculate $s_{L P}\left(t_{2}\right)$, we note that $L P$ becomes active at $t=t_{1}$. Thus independent of the value of $s_{L P}$ prior to $t_{1}, s_{L P}\left(t_{1}\right)=d_{\max , L P}$, where

$$
d_{m u x, L P}\left(P, T_{L P}\right)=\widehat{d}_{L P}\left(P, T_{L P}\right) \frac{\left[1-e^{-\left(P-T_{L P}\right) / \tau_{c}}\right]}{1-e^{-T_{L P} / \tau_{d}} e^{-\left(P-T_{L P}\right) / \tau_{c}}} .
$$

$L P$ remains active until time $t_{2}$ which implies

$$
s_{L P}\left(t_{2}\right)=d_{\max , L P} e^{-\left(t_{2}-t_{1}\right) / \tau_{4}}
$$

Similar to before $w_{P Y}\left(t_{2}\right)=\hat{w}_{P Y} \exp \left(-t_{2} / \tau_{w, P Y}\right)$ where $\tau_{w, P Y}$ is the time constant for $P Y$ activity in its silent state. We can now substitute into (21) to obtain the following two equations for $t_{1}$ and $t_{2}$ :

$$
\begin{aligned}
& \bar{g}_{A B} d_{\text {max } x A B}(P) e^{-T_{A B} / \tau_{\zeta}} e^{-\left(t_{1}-T_{A B}\right) / \tau_{\kappa}}+\bar{g}_{P Y} d_{\text {max }, P Y}\left(P, T_{P Y}\right) e^{-T_{P Y} / \tau_{2}} e^{-t_{1} / \tau_{1}} \\
& +M_{1} \widehat{w}_{L P} e^{\left.-\left(P-T_{L P}\right)\right) / \tau_{w, L P}}=g_{s y n 1}^{*} . \\
& \quad \bar{g}_{A B} d_{\text {max }, A B}(P) e^{-T_{A B} / \tau_{\zeta}} e^{-\left(t_{2}-T_{A B}\right) / \tau_{\kappa}}+\bar{g}_{L P} d_{\text {max }, L P}\left(P, T_{L P}\right) e^{-T_{L P} / \tau_{4}} \\
& \quad+M_{2} \widehat{w}_{P Y} e^{-t_{2} / \tau_{w, P Y}}=g_{s y n 2}^{*} .
\end{aligned}
$$

Note that $T_{P Y}=P-t_{2}$, and if $\bar{g}_{P Y}=0$ then $T_{L P}=P-t_{1}$, while if $\bar{g}_{P Y}>0$ then $T_{L P}=t_{2}-t_{1}$.

Equations (25) and (26) can be solved numerically to obtain $t_{1}$ and $t_{2}$. In specific parameter regimes, certain terms on the left-hand side of the above equations become small and an analytic estimate for $t_{1}$ and $t_{2}$ becomes possible. Our goal for the remainder of the paper is to use (25) and (26) to understand how $\varphi_{L P}=t_{1} / P$ and $\varphi_{P Y}=t_{2} / P$ depend on the parameters in these equations. We shall check this for several cases; when the $L P$ and $P Y$ inhibition is absent (section 3.1), when $L P$ to $P Y$ inhibition is added (section 3.2), when $P Y$ to $L P$ inhibition is present (section 3.3), and when both reciprocal synapses are present (section 3.4).

\subsection{Phase determination with no synapses between $L P$ and $P Y$}

When the synapses between $L P$ and $P Y$ are removed, we set $\bar{g}_{L P}=0$ and $\bar{g}_{P Y}=0$ in equations (25) and (26). Then $t_{1}$ and $t_{2}$ (and, therefore $\varphi_{L P}$ and $\varphi_{P Y}$ ) are determined by the properties of the $A B$ synapses and the intrinsic properties of the neurons. In this case, the dependence of $\varphi_{L P}$ on $P$ has been previously shown by Manor et al [17], and that of $\varphi_{P Y}$ is similar.

If the $A B$ synapses onto $L P$ and $P Y$ are not depressing, then $\varphi_{L P}$ and $\varphi_{P Y}$ both decay like $1 / P$ since (25) and (26) are no longer period dependent. For each cell, this decay is controlled either by $\tau_{\kappa}$ the time constant of decay of the $A B$ synapses and $\bar{g}_{A B}$ the maximal conductance of the synapse or by the cell's own intrinsic time constant of decay in the silent state, $\tau_{w, L P}$ or 
$\tau_{w, P Y}$. The situation changes dramatically when the $A B$ synapses are depressing. Following the results of Manor et al [17], with an appropriate choice of parameters, both sets of parameters can control the phase curves, each doing so at different ranges of periods. In particular, when $\tau_{\kappa}$ is large enough so that the synaptic decay is slower than the rate at which $w_{P Y}$ and $w_{L P}$ decay on the left branches of their respective nullclines, phase maintenance is enhanced. In Fig. 5A, we can see that for $500<P<650, \varphi_{L P}$ and $\varphi_{P Y}$ decrease. In this range of periods, the synapses from $A B$ to $L P$ and $P Y$ are largely depressed and, consequently, do not significantly contribute to the time at which $L P$ and $P Y$ fire. Here the first terms on the left-hand sides of (25) and (26) are close to zero, the second terms are absent $\left(\bar{g}_{P Y}=\bar{g}_{L P}=0\right)$ and therefore the third terms alone determine $t_{1}$ and $t_{2}$. Thus in this range, phase is mostly determined by the intrinsic dynamics of $L P$ and $P Y$. In the range $650<P<1000$, the $A B$ synapse increasingly recovers from depression $\left(d_{\max , A B}\right.$ gets larger; Fig $\left.5 \mathrm{~B}\right)$. As a result the synaptic properties take over control for setting $\varphi_{L P}$ and $\varphi_{P Y}$ in this range. For $P>1000$, the $A B$ synapse has maximally recovered from depression causing $d_{\max , A B}$ to saturate. Now the first terms on the left-hand sides of (25) and (26) take longer to decay then the third terms (since $\tau_{\kappa}$ is large enough). Thus the synapses from $A B$ determine the firing times. However, as the period increases, the phase decreases like $1 / P$ since the value of $d_{\max , A B}$ in the first terms will eventually saturate;

$d_{\max , A B} \rightarrow 1$ as $P \rightarrow \infty$ [17]. These three regimes combine together to cause the phase curve to be cubic in shape. Thus, although a constant phase is not perfectly achieved, phase-maintenance is better than when the $A B$ synapse is non-depressing where $\varphi_{L P}$ and $\varphi_{P Y}$ decay like $1 / P$.

\subsection{The effect of $L P$ to $P Y$ inhibition on $\varphi_{P Y}$}

We now explore how the presence of the $L P$ to $P Y$ synapse affects $\varphi_{P Y}$. We show that the inclusion of the $L P$ to $P Y$ synapse leads to another way to control the phase of $P Y$ activity. In (25) and (26), we let $\bar{g}_{P Y}=0$ while setting $\bar{g}_{L P}$ to a positive value. When the synapse from $L P$ to $P Y$ is present, each time $L P$ is active, it provides further inhibition to $P Y$. In order for $P Y$ to fire, the $L P$ inhibition must also decay. Therefore, the additional time it now takes for $P Y$ to fire is determined, in part, by the the size of $\bar{g}_{L P} s_{L P}$ and the rate at which $s_{L P}$ decays. Using equation (26), we can determine over which parameter regimes the $L P$ to $P Y$ synapse significantly contributes to $\varphi_{P Y}$. For example, suppose $\tau_{4}$, the time constant of decay of the $s_{L P}$ synapse when $L P$ is active, is small enough, or that $\bar{g}_{L P}$, the maximal conductance of this synapse is small. Then the term $\bar{g}_{L P} s_{L P}$ is insignificant and, therefore the $L P$ to $P Y$ synapse does not significantly affect $t_{2}$. However, if $\tau_{4}$ is not so small compared with $\tau_{K}$ and $\tau_{w, P Y}$, and if $\bar{g}_{L P}$ is big enough, then the $L P$ inhibition will more significantly contribute to $\varphi_{P Y}$

The period dependence of the $L P$ synapse and the rates of depression and recovery also play a role in setting phase. For example if the time constant of recovery from depression of the $L P$ synapse $\left(\tau_{c}\right)$ is very small, then the synapse will recover quickly and will largely be period independent. Similarly, if the depression time constant $\tau_{d}$ is small, then the synapse will be ineffective at suppressing its post-synaptic target. More interestingly, depending on the value of parameters, the the $L P$ to $P Y$ synapse can work in concert with or independently of the $A B$ synapse to effect $P Y$ firing. Figure 6A shows graphs of the $\varphi_{L P}, \varphi_{P Y}$ and $d_{\max , L P}$ for different choices of the parameter $P_{2}$, the half activation of the strength of the $L P$ to $P Y$. The dashed line traces show the case when $P_{2}=570$ (note the half-activation of the $A B$ synapse $P_{1}=870$ in all cases), the dotted line traces when $P_{2}=870$ and the solid traces when $P_{2}=1770$. In the latter case, the length of the $L P$ interburst $\left(t_{1}=P-T_{L P}\right)$ never becomes large enough to cause the $L P$ to $P Y$ synapse to gain strength. Thus $d_{\max , L P}=0$ (Fig. 6B) and $\varphi_{P Y}$ looks as it would if $\bar{g}_{L P}=0$. When the parameter $P_{2}$ is lowered to 870 , then the $L P$ to $P Y$ synapse does strengthen around $P=1380$ (dotted traces) because $P-T_{L P}$ becomes larger than $P_{2}$. Here, the synapse becomes strong in a range of periods for which the $A B$ synapse is already saturated. Thus it controls $\varphi_{P Y}$ quite independently of the $A B$ to $P Y$ synapse. When, $P_{2}=190$ (dashed traces), the $L P$ to $P Y$ synapse works in concert with the $A B$ to $P Y$ synapse to delay $P Y$ firing. Here the 
phase curve has a single local maximum that is larger in value than when the $L P$ to $P Y$ synapse is absent. Numerical simulations of the full set of equations using XPPAUT yield similar results (not shown). As $P \rightarrow \infty$, the value of $d_{\max , L P}$ tends to $d{ }^{\wedge}{ }_{L P}\left[1-\exp \left(-\left(P-T_{L P}\right)=\tau_{c}\right)\right]$ since $T_{L P} \rightarrow \infty$ as can be seen from equations (14) and (23). Thus $d_{\max , L P}$ is bounded away from one and thus the $L P$ to $P Y$ synapse never fully recovers.

\subsection{The role of $P Y$ to $L P$ inhibition on $\varphi_{L P}$ and $\varphi_{P Y}$}

Next we address what effect the synapse from $P Y$ to $L P$ has on the phase of $L P$ and $P Y$. Again depending on the parameters, there are many possibilities that can be considered. However we shall take a cue from the biological system and study one important subcase. It is known that the $P Y$ to $L P$ synapse is initially strong, depresses very quickly and also decays very quickly once $P Y$ becomes silent [16]. From these results, we conclude that the primary role of the $P Y$ to $L P$ synapse is to end the firing of $L P$ and return it to its active state. This simple role, however, has a dramatic affect on both $\varphi_{L P}$ and $\varphi_{P Y}$. First, the knocking down of $L P$ to its silent state by $P Y$ means that at small periods, $\varphi_{L P}$ decreases. In particular, for small values of $P$, the $A B$ synapse is weak and does not play a big role in setting $t_{1}$, which is set mainly by the intrinsic properties of $L P$. Once $L P$ is on the left branches of its $v_{L P}$ nullcline, $w_{L P}$ begins to decay before $A B$ becomes active. Thus when $A B$ next becomes active, $L P$ is much closer to the jump surface than it would have been had it been knocked down to the silent state by $A B$, Fig. 7. Thus the remaining time that $L P$ must now spend evolving toward the jump surface is much smaller in the presence of the $P Y$ synapse than in its absence. Therefore $t_{1}$ is significantly smaller and $\varphi_{L P}$ decreases. At large $P$, however, the $A B$ synapse is stronger and continues to dominate the determination of $t_{1}$. This is especially true if we choose $\tau_{1}$, the time constant of decay of the $P Y$ to $L P$ inhibition to be small.

The second effect of the presence of the $P Y$ to $L P$ synapse is to strengthen the $L P$ to $P Y$ synapse. This is because the presence of the $P Y$ to $L P$ synapse causes $L P$ to have a shorter active duration than when this synapse is absent. Note that when $\bar{g}_{P Y}=0$ (the $P Y$ to $L P$ inhibition is absent), $T_{L P}=P-t_{1}$, whereas when $\bar{g}_{P Y}>0$ (the $P Y$ to $L P$ inhibition is present), $T_{L P}=t_{2}-t_{1}$ because the $P Y$ inhibition ends the $L P$ burst. Thus $L P$ spends a larger fraction of its period in the silent state, which, in turn increases both terms of equation (23) allowing $d_{\max , L P}$ to increase with $P$, Fig. 8. Moreover, since $T_{L P}$ is bounded as $P \rightarrow \infty, d_{\max , L P} \rightarrow 1$ in this limit. Therefore independent of the value of $P_{2}$, the $L P$ to $P Y$ synapse will play a role in setting the $P Y$ phase. Thus, the effect of $P Y$ inhibition is to strengthen the inhibition that it itself receives! The ramifications of this are explored in the next section.

\subsection{Reciprocal inhibition between $L P$ and $P Y$}

The above results indicate that the inhibitory synapses between the two pyloric neurons $L P$ and $P Y$ can play a role in enhancing the ability of the neurons to achieve phase maintenance. Indeed, we have seen that the $L P$ to $P Y$ synapse can control $P Y$ phase in a range of periods for which the $A B$ synapses to $L P$ and $P Y$ are already saturated, and thus are no longer period dependent. Therefore, it is natural to consider cases in which the $A B$ inhibition is both nondepressing and depressing.

When the $A B$ synapse is non-depressing $\left(d_{\max , A B} \equiv 1\right)$, and the inhibition between $L P$ and $P Y$ is absent, $\varphi_{L P}$ and $\varphi_{P Y}$ decrease like $1 / P$. In order for the synapse from $L P$ to $P Y$ to be able to create a range of periods over which $\varphi_{P Y}$ increases, $d_{L P}$ must increase as the period increases. Similarly, $d_{P Y}$ must increase as the period increases in order to create a range of periods over which $\varphi_{L P}$ increases. Let us first consider $d_{\max , L P}$ as given by equation (23). This term will increase as period increases so long as $L P$ spends increasingly longer times in the silent state. As $P$ increases, even though the time $t_{1}$ need not increase the time $P-T_{L P}$ will. To understand this, recall that one role of the $P Y$ inhibition to $L P$ is to return the latter to the silent state at an 
earlier stage than $A B$ would have. This is independent of depression of either the $A B$ or $P Y$ synapses. Thus the $L P$ to $P Y$ synapse will have a chance to recover for more time and thereby will strengthen. A stronger $L P$ to $P Y$ synapse delays $P Y$ firing and thus increases $\varphi_{P Y}$. In Fig 9A, we show an example of this; panel A1 is obtained by solving the full set of equations numerically with XPPAUT, while panel A2 is obtained by solving (25) and (26) with MATLAB. In particular, the $L P$ to $P Y$ synapse becomes strong when $P-T_{L P}>P_{2}(=1140)$. In this range, the value of the synaptic and depression time constants associated with the $L P$ synapse play an increasingly larger role in setting $P Y$ phase. If the time constant of $L P$ to $P Y$ synaptic decay is chosen to be large relative to the time constant of decay of the $A B$ synapse, then the $L P$ synapse can increase the phase of $P Y$ for a range of periods larger than $P_{2}$. For very large values of $P$, the $L P$ synapse will fully recover from depression and will not be able to further increase the delay of $P Y$ firing. Thus for large $P$, we expect $\varphi_{P Y}$ to decay like $1 / P$ as shown in the figure.

A delay in $P Y$ firing has the added effect of strengthening the $P Y$ to $L P$ synapse provided that this synapse is also depressing, as $P Y$ spends more time in the silent phase. This can have a very subtle effect on the $L P$ phase. At first glance, the $P Y$ to $L P$ synapse does not appear to do much to help $L P$ phase be constant, instead almost all of this work falls on $A B$. However $P Y$ does put $L P$ in a position so that at small periods, the intrinsic controlled $L P$ phase is much closer to the larger period $A B$-synaptically controlled $L P$ phase. Moreover at these larger periods where the $A B$ synapse is strengthening, increases in strength of the $P Y$ to $L P$ synapse can induce changes in the $L P$ phase. This is because this synapse can now complement the $A B$ to $L P$ synapse to keep the $L P$ neuron near the jump surface for a longer time, thereby delaying $L P$ firing. This subtle effect, however, does not dramatically affect the $L P$ phase since the time constant of decay of the $P Y$ to $L P$ synapse is small. Thus its effect will have worn off by the next $L P$ cycle. This is consistent with the biological system where the $P Y$ to $L P$ synapse is a strong, short lasting one whose main effect is to terminate $L P$ firing [16].

In summary, when the $A B$ input is non-depressing, the depression of the $L P$ to $P Y$ synapse can still be utilized to improve phase maintenance of $P Y$. However the phase of $L P$ is still mostly determined by the $A B$ synapse and basically decays like $1=P$.

When the reciprocal inhibition between $L P$ and $P Y$ is present and the $A B$ synapses are depressing, the complexity of the network is increased. However, the dynamics of $L P$ and $P Y$ can be understood based on the results of sections 3.1-3.3. As discussed above, depending on parameters, various synaptic or intrinsic parameters play a role in setting phase at different intervals of period. Let us focus on the case where $P_{2}>P_{1}\left(P_{2}=1470\right.$ and $\left.P_{1}=870\right)$. In this case, both $\varphi_{L P}$ and $\varphi_{P Y}$ can have several local maxima and minima and, in particular, can have good phase maintenance over a large range of periods. Figure 9B shows one such case where $\varphi_{L P}$ varies between .35 and .45 and $\varphi_{P Y}$ varies between .6 and .7 for $P 2$ [500; 2400]. These curves can be parsed into three distinct intervals. At small periods, the $A B, L P$ and $P Y$ synapses are weak and play little role in setting phase. Here intrinsic parameters of $L P$ and $P Y$, namely $\tau_{w, L P}$ and $\tau_{w, P Y}$, are more important; only the last term of the left hand side of equations (25) and (26) is large. At intermediate periods, the $A B$ synapse strengthens while the $L P$ and $P Y$ synapses remain weak. Here, the time constant of $A B$ synaptic decay, $\tau_{k}$, is the most important parameter in setting phase. The first term on the left hand side of (25) and (26) is dominant. Finally, at larger $P$, the $A B$ synapse has saturated and plays no additional role in setting phase. Instead, at these periods the $L P$ and $P Y$ synapses have strengthened enough to be important. In particular, the inhibition from $P Y$ to $L P$ allows $L P$ to spend more time in the silent state, thereby strengthening the $L P$ to $P Y$ synapse. This strengthened synapse in turn delays $P Y$ firing, increasing its phase, and strengthening the $P Y$ to $L P$ synapse. The time constants of decay between the $L P$ and $P Y$ reciprocal synapses play the most important role of setting phase for large $P$. In this case, the first and second terms on the left hand side of (25) and (26) are large, 
but the first terms decay more quickly then the second terms. Thus the control of phase for large $P$ is most strongly controlled by time constants associated with the reciprocal $L P$ and $P Y$ synapses.

The importance of the $P Y$ to $L P$ synapse in redirecting the role of the $L P$ to $P Y$ synapse can not be overstated. In particular, when the $P Y$ to $L P$ synapse is absent, the $L P$ to $P Y$ synapse strengthens only as a result of the effect of the delay to firing induced by the $A B$ to $L P$ synapse. The $L P$ to $P Y$ synapse is only effective in a range of periods that depends closely on the $A B$ to $L P$ synapse; see section 3.2 and the relationship between the parameters $P_{1}$ and $P_{2}$. However, when the $P Y$ to $L P$ synapse is present, the $L P$ to $P Y$ synapse can act much more independently. Namely, it can strengthen in a range of periods that is largely independent of any effects of the $A B$ induced delay, and therefore can affect the $P Y$ phase curve in a range of periods that is different than the $A B$ synapse does. In particular, in order for the $L P$ synapse to strengthen, the value of $P_{2}$, the half-activation of the depression and recovery term associated with $d_{L P}$ need not be related to $P_{1}$, the half-activation associated with $d_{A B}$.

\section{Discussion}

Central pattern generating (CPG) networks are often driven by pacemaker neurons that set the network frequency and the influence the firing times of follower neurons via feed-forward synapses [7]. Nonetheless, the dynamics of these networks are often modulated or controlled by mechanisms that are downstream from the feed-forward inputs. Even in small networks, the dynamics resulting from the interactions among the rhythmically active neurons can be surprisingly complicated. We have analyzed a simplified network, modeling the pyloric CPG of the crustacean stomatogastric ganglion. Our results build on our previous studies that examined the effect of short-term synaptic depression, a common property of many synapses, on the activity phase of a follower neuron in a two-cell feed-forward network [4,17].

In this study we focused on a three-cell network, consisting of a pacemaker neuron $A B$ that produces feed-forward inhibitory synapses on two follower neurons $L P$ and $P Y$. The follower neurons also have reciprocally-inhibitory synaptic connections thus producing feedback interactions in the network. We examined the mechanisms that determine the activity phase of $L P$ and $P Y$ in face of changes in network frequency. Our analysis demonstrates that phase constancy is enhanced when the synapses between neurons are depressing. As a result, the $P Y$ and $L P$ neurons can keep a relatively constant phase over a four-fold change in the network cycle period, consistent with the experimental findings of Hooper [13].

Mathematical analysis carried out in lower dimensional phase spaces allowed us to understand how different combinations of parameters can affect the activity phase of follower neurons in different ranges of the cycle period. It also revealed a number of interesting facts about how the dynamics of the network evolve. For example, it has traditionally been thought that the primary role of the $P Y$ to $L P$ synapse is simply to return $L P$ to its silent state [27]. Our analysis now demonstrates that an additional effect of this synapse is to strengthen the $L P$ to $P Y$ synapse by causing $L P$ to spend more time in its silent state, allowing for recovery from short-term depression. This effect occurs whether or not the $P Y$ to $L P$ synapse is depressing, thus indicating that the plasticity of this synapse may not be so critical to the proper functioning of the network.

\subsection{Role of synaptic depression}

Many synaptic connections in CPGs exhibit short-term depression [19,23,26]. Our results show that synaptic depression can play a variety of potential roles in setting the phases of the follower $L P$ and $P Y$ neurons. First, the feed-forward synapses from $A B$ to both $L P$ and $P Y$ allow the intrinsic properties of these follower neurons to determine phase at small periods where the $A B$ synapses are weak. At intermediate cycle periods, these same synapses strengthen and 
become more effective in the control of the activity phases. Second, the depression in the $L P$ to $P Y$ synapse allows the $P Y$ phase to be determined by this synapse in different ranges of periods. If the $L P$ synapse strengthens in the same range of periods as the $A B$ synapses, then these two work in concert to determine $P Y$ phase. If not, the $L P$ to $P Y$ synapse can work independently of the $A B$ to $P Y$ synapse to influence the phase of $P Y$ activity. As a consequence, even if the $A B$ synapse is non-depressing, the $L P$ to $P Y$ synapse can still promote phase constancy of $\varphi_{P Y}$ (to be better than $1 / P$ ) in some range of periods. Third, as shown in section 3.4 , depression in the $P Y$ to $L P$ synapse can also have a small but subtle effect on the $L P$ phase. Thus, changes in the cycle period of the pacemaker neuron $A B$ may not directly affect $A B$ 's synapses, yet result in changes in downstream synapses that the pacemaker does not directly target. This provides another set of candidate mechanisms that can affect the phase of the follower neurons.

\subsection{Consequences of model assumptions}

The primary modeling assumption made here is that the individual spikes within a burst are not of large relevance in determining the phase relationships of neurons. Synapses in the stomatogastric ganglion have both a spike-mediated and a non-spike-mediated (graded) component whose strength depends on the slow oscillatory waveforms of the presynaptic neuron [12]. When action potentials, and therefore spike-mediated transmission, is blocked by bath application of tetrodotoxin, a tri-phasic rhythm characteristic of the pyloric network activity can be generated by applying various modulatory substances [2]. Thus, the simplified Morris-Lecar type models and graded synapses used provide a good first-order approximation of the activity of the pyloric network, in particular the envelope of the slow oscillations (as in Fig. 1) and its underlying synaptic mechanisms. We note that our model of short-term depression was chosen to match known biological results. In particular, equations (13) and (14) are used specifically to model the longer time scale associated with recovery of pyloric synapses $[3,28]$. The specific form of these synapses is important to our results in that changes to parameters associated with these equations (as in Fig 6) may lead to deterioration of phase maintenance. We also chose the time constants of the various synaptic connections to mimic known biological facts. For example, the time constants associated with the $P Y$ to $L P$ synapse were chosen to make it strong, fast decaying and fast depressing consistent with published experimental results [16].

The other major modeling assumption is that the jump surfaces in the silent states of $L P$ and $P Y$ are planes. This assumption allowed us to analytically derive (25) and (26). The results shown in Fig. 9 validate this assumption as they show a very good qualitative agreement between phase curves obtained from (25) and (26) with those obtained by numerically solving the full set of equations. This qualitative agreement occurs despite the fact that our analytic reduction effectively ignores the behavior of the neurons when they are active and only records their $w$ values at the moment they return to the silent state.

In our earlier work [17], we considered cases where $P$ was varied either by increasing $T_{A B}$ while keeping $T_{\text {inactive }}$ fixed or by increasing both $T_{A B}$ and $T_{\text {inactive }}$ but keeping the duty cycle $\left(T_{A B} / P\right)$ constant. We showed that changing the period in the feedforward $A B-L P$ network in either of these ways still allows the phase of $L P$ to be controlled by either synaptic or intrinsic parameters at different ranges of periods. In the current model, our derived formulas (25) and (26) allow us to again consider either of those two possibilities. The same qualitative behavior exists in both cases, namely that there are distinct ranges of period over which one set of parameters or the other would dominate phase determination. However, the shapes of the phase curves and their dependence on various parameters are quantitatively different in the two cases. 


\subsection{Conclusions}

Many neural networks maintain a constant relative phase in the activity patterns of participating neurons despite changes in network frequency [6,25]. In CPGs this constraint on phase is of special importance because the underlying neural patterns are commanded to muscles that often have to be activated at precise phases of each cycle in order to produce meaningful behavior, for example, locomotion $[29,30]$. We have built on our previous modeling results to propose mechanisms through which synaptic dynamics, such as short-term depression, can contribute to phase maintenance in face of changes in network frequency. Our most important findings are that synaptic interactions among follower neurons may affect their phase in each cycle of oscillation and that these interactions may extend the range of cycle periods for which there is phase maintenance beyond those made possible by the dynamics of the feed-forward synapses from the pacemaker neurons. These predictions are potentially important for understanding mechanisms underlying phase maintenance in the pyloric network and can be experimentally tested by manipulating the strength and dynamics of the synapses in this network using pharmacological agents or the dynamic clamp technique.

\section{Acknowledgements}

This work was supported, in part, by a grant from The City University of New York PSC-CUNY Research Award Program (CM), the National Science Foundation DMS-0315862 (AB, CM) and DMS-0615168 (AB) and the National Institutes of Health MH-60605 (FN).

\section{References}

1. Ambrosio-Mouser C, Nadim F, Bose A. The effects of varying the timing of inputs on a neural oscillator. SIAM J Appl Dyn Syst 2006;5:108-139.

2. Anderson WW, Barker DL. Synaptic mechanisms that generate network oscillations in the absence of discrete postsynaptic potentials. J Exp Zool 1981;216:187-191. [PubMed: 7288387]

3. Birmingham, JT.; Manor, Y.; Nadim, F.; Abbott, LF.; Marder, E. An empirical model describing the dynamics of graded synaptic transmission in the lobster pyloric network. In: Bower, James M., editor. Computational Neuroscience: Trends in Research. Plenum Press; NY: 1998.

4. Bose A, Manor Y, Nadim F. The activity phase of postsynaptic neurons in a simplified rhythmic network. J Comput Neurosci 2004;17:245-261. [PubMed: 15306742]

5. Cohen A, Ermentrout GB, Kiemel T, Kopell N, Sigvardt K, Williams T. Modelling of intersegmental coordination in the lamprey central pattern generator for locomotion. TINS 1992;15:434-438. [PubMed: 1281350]

6. DiCaprio RA, Jordan G, Hampton T. Maintenance of motor pattern phase relationships in the ventilatory system of the crab. J Exp Biol 1997;200:963-974. [PubMed: 9318763]

7. Dickinson PS. Neuromodulation of central pattern generators in invertebrates and vertebrates. Curr Opin Neurobiol 2006;16:604-614. [PubMed: 17085040]

8. Ermentrout GB. n:m phase-locking of weakly coupled oscillators. J Math Biol 1981;12:327-342.

9. Ermentrout GB. Simulating, analyzing, and animating dynamical systems: A guide to XPPAUT for researchers and students. SIAM. 2002

10. Friesen WO, Pearce RA. Mechanisms of intersegmental coordination in leech locomotion. Sem Neurosci 1993;5:41-47.

11. Grillner, S. Control of locomotion in bipeds, tetrapods and fish. In: VBB, editor. Handbook of Physiology, section 1, The Nervous system. II. Maryland: Waverly Press; 1981. p. 1179-1236.

12. Graubard K, Raper JA, Hartline DK. Graded synaptic transmission between identified spiking neurons. J Neurophysiol 1983;50:508-521. [PubMed: 6136554]

13. Hooper S. Phase maintenance in the pyloric pattern of the lobster (Panulirus interruptus) stomatogastric ganglion. J Comput Neurosci 1997;4:191-205. [PubMed: 9257232]

14. Hooper S. The pyloric pattern of the lobster (Panulirus interruptus) stomatogastric ganglion comprises two phase-maintaining subsets. J Comput Neurosci 1997;4:207-219. [PubMed: 9257233] 
15. Jones S, Kopell N. Local network parameters can affect inter-network phase lags in central pattern generators. J Math Biol 2006;52:115-40. [PubMed: 16195924]

16. Mamiya A, Manor Y, Nadim F. Short-term dynamics of a mixed chemical and electrical synapse in a rhythmic network. J Neurosci 2003;23:9957-9564.

17. Manor Y, Bose A, Booth V, Nadim F. Contribution of synaptic depression to phase maintenance in a model rhythmic network. J Neurophysiol 2003;90:3513-3528. [PubMed: 12815020]

18. Marder E, Calabrese R. Principles of rhythmic motor pattern generation. Physiol Rev 1996;76:687717. [PubMed: 8757786]

19. Marder E. From biophysics to models of network function. Annu Rev Neurosci 1998;21:25-45. [PubMed: 9530490]

20. Marder E, Bucher D. Understanding circuit dynamics using the stomatogastric nervous system of lobsters and crabs. Annu Rev Physiol 2006;69:291-316. [PubMed: 17009928]

21. Mishchenko, EF.; Rozov, NK. Differential Equations with Small Parameters and Relaxation Oscillators. New York: Plenum Press; 1980.

22. Morris C, Lecar H. Voltage oscillations in the barnacle giant muscle fiber. Biophys J 1981;35:193213. [PubMed: 7260316]

23. Nadim F, Manor Y, Kopell N, Marder E. Synaptic depression creates a switch that controls the frequency of an oscillatory circuit. PNAS 1999;96:8206-8211. [PubMed: 10393973]

24. Nusbaum MP, Beenhakker MP. A small-systems approach to motor pattern generation. Nature 2002;417:343-50. [PubMed: 12015615]

25. O'Keefe J, Recce ML. Phase relationship between hippocampal place units and the EEG theta rhythm. Hippocampus 1993;3:317-330. [PubMed: 8353611]

26. Parker D, Grillner S. Activity-dependent metaplasticity of inhibitory and excitatory synaptic transmission in the lamprey spinal cord locomotor network. J Neurosci 1999;19:1647-1656. [PubMed: 10024351]

27. Rabbah P, Nadim F. Synaptic dynamics do not determine proper phase of activity in a central pattern generator. J Neurosci 2005;25:11269-11278. [PubMed: 16339022]

28. Rabbah P, Nadim F. Distinct synaptic dynamics of heterogeneous pacemaker neurons in an oscillatory network. J Neurophsyiol 2007;97:2239-2253.

29. Sigvardt KA. Spinal mechanisms in the control of lamprey swimming. Am Zool 1981;29:19-35.

30. Skinner F, Mulloney B. Intersegmental coordination of limb movements during locomotion:

Mathematical models predict circuits that drive swimmeret beating. J Neurosci 1998;18:3831-3842.

[PubMed: 9570812]

5 Appendix

We numerically solved equations (1)-(14) to obtain our results. In these equations $m_{\infty}\left(v_{x}\right)=0.5$ $\left(1+\tanh \left(\left(v_{x}+1.2\right) / 18\right)\right), w_{\infty}\left(v_{x}\right)=0.5\left(1+\tanh \left(\left(v_{x}-15\right) / 5\right)\right)$, and $\tau_{w}\left(v_{x}\right)=m_{x}\left(40-30 w_{\infty}\left(v_{x}\right)\right)$. For both $L P$ and $P Y, I_{x}=75, g_{\text {leak }}=2, E_{\text {leak }}=-60, E_{\text {inh }}=-80, g_{C a}=4, E_{C a}=120, g_{K}=8$, and $E_{K}=-84$. In all simulations, $T_{A B}=300, \tau_{\alpha}=1800$, and $\tau_{\beta}=15$.

In the case of the depressing synaptic $A B$ input, $m_{L P}=8.1, m_{P Y}=8.4$ and $\bar{g}_{A B}=1.8$. When the $L P$ and $P Y$ synaptic inputs were present, we set $\bar{g}_{L P}=1$ and $\bar{g}_{P Y}=2$. However, when these synapses were not present, $\bar{g}_{L P}$ and $\bar{g}_{P Y}$ were set equal to 0 . The remaining parameter values were as follows : $\tau_{k}=1650, \tau_{c}=3300, \tau_{d}=990, \tau_{a}=2700, \tau_{b}=150, \tau_{1}=60, \tau_{2}=600, \tau_{3}=$ $210, \tau_{4}=300, x_{1}=55, x_{2}=35, P_{1}=570$ and $P_{2}=1470$.

When the $A B$ synapses were not depressing, we simply set $d_{A B} \equiv 0.38$. We changed $m_{L P}=$ $2.55, m_{P Y}=3.15, \bar{g}_{A B}=1.4, \bar{g}_{L P}=13, \bar{g}_{P Y}=11, \tau_{k}=1200, \tau_{c}=60, \tau_{d}=60, \tau_{a}=1350, \tau_{b}=$ $240, \tau_{1}=60, \tau_{2}=1350, \tau_{3}=330, \tau_{4}=60, P_{2}=1140$ and $x_{2}=10$.

We solved equations (25) and (26) using MATLAB. We used the same set of parameter values as we did when using XPPAUT. There are a few parameters in these equations that do not explicitly appear in (1)-(14) that can be estimated from the geometry of the nullclines. We set $M_{1}=M_{2}=3.12, \hat{w}_{L P}=\hat{w}_{P Y}=1$. For the non-depressing case Fig. 9 A1 and A2, 
$g_{s y n 1}^{*}=2, g_{\text {syn } 2}^{*}=1.5, \tau_{w, L P}=102, \tau_{w, P Y}=126$, and for the depressing case Fig. 9, B1 and B2, $g_{\text {syn } 1}^{*}=1.05, g_{\text {syn } 2}^{*}=1, \tau_{w, L P}=240, \tau_{w, P Y}=255$. 


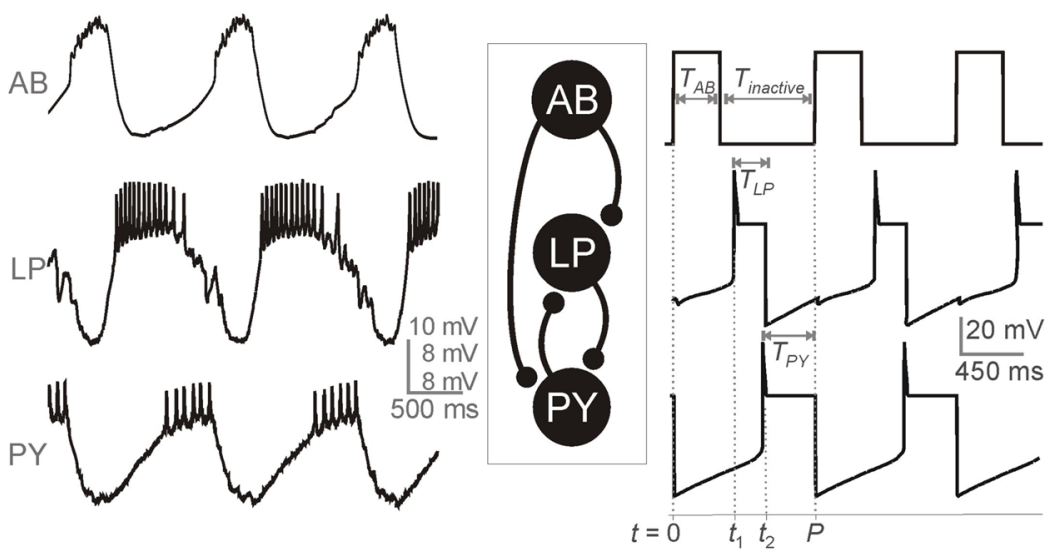

Fig. 1.

Voltage traces of a typical ongoing tri-phasic pyloric rhythmic activity involving the pacemaker neuron $A B$ and follower neurons $L P$ and $P Y$. Left traces show experimental recordings (Nadim, unpublished data) and the traces on the right are the simulation of our model which provides an approximation to the envelope of oscillations observed in the experimental traces. The temporal parameters are marked in the model traces. The middle panel is a reduced schematic diagram of the pyloric network, showing the cells and synapses used in the model. 


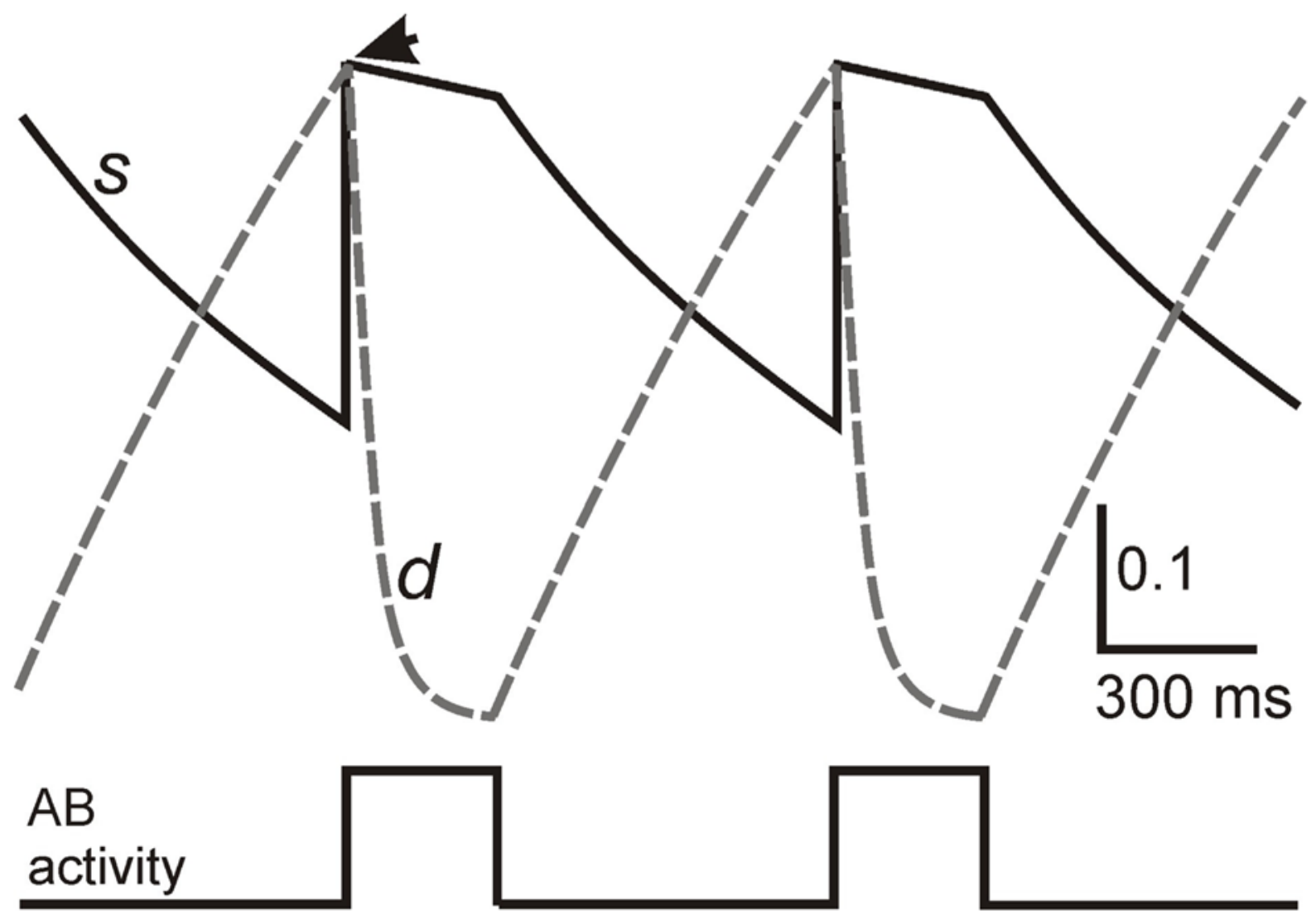

Fig. 2.

Dynamics of the synaptic and depression variables. A schematic associated with the $\mathrm{AB}$ synapse is shown. At the moment (marked by arrow) $A B$ becomes active, the $s$ variable (solid) is reset to the current value of $d$ (dashed). At all other times, the two variables are decoupled and governed by distinct sets of equations. During the time $A B$ is active, $s$ decays at a slow rate and $d$ depresses. Once $A B$ becomes inactive, $s$ decays at a different rate, while $d$ recovers. The $s$ and $d$ variables for the $L P$ and $P Y$ synapses behave in a similar manner. 

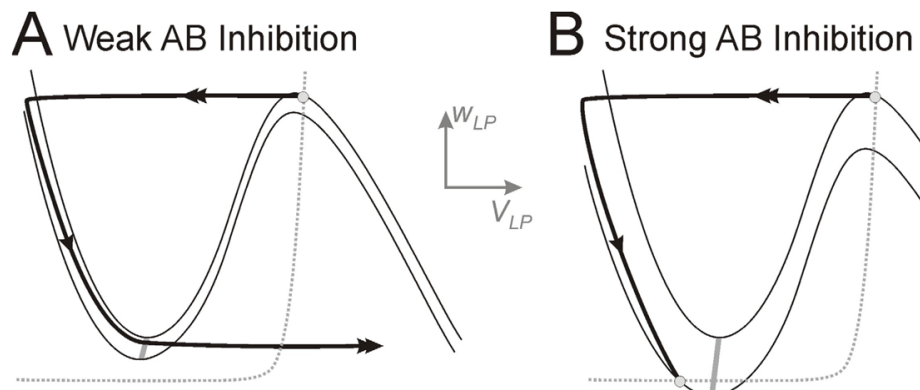

Fig. 3.

The influence of $A B$ inhibition on the dynamics of $L P$.A. The inhibition from $A B$ to $L P$ causes $L P$ to return to the silent state, but is too weak to affect the time that $L P$ spends in this state. Note the lack of intersection of the $v$ - (solid) and $w$-nullclines (dotted) along the left branches. B. The inhibition from $A B$ is strong and creates a fixed point on the left branch of the $v$-nullcline. In this case $L P$ must wait for $A B$ inhibition to decay, thereby allowing the stable fixed point (lower white circle) to disappear before it can jump to the active state. White circles indicate stable fixed points. The two $v$-nullclines in each case indicate no (upper) or maximal (lower) inhibition from $A B$ to $L P$. The gray curve is the $L P$ jump curve. 

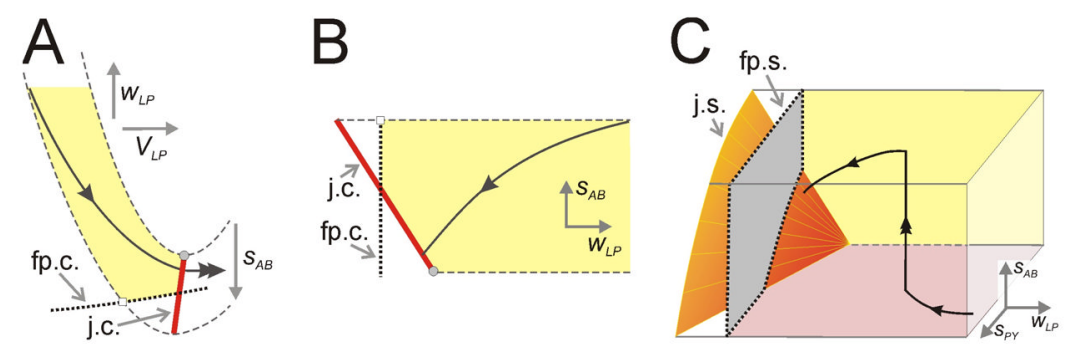

Fig. 4.

Jump curves and surfaces. A. The dynamics of $L P$ activity in response to $A B$ inhibition alone. The set of local minima of the $v$-nullclines forms a one-dimensional jump curve j.c. The $v$ nullclines for different values of $\mathrm{AB}$ inhibition $\left(s_{A B}\right)$ form a surface (shaded) that is bounded by two extremes (dashed) in each case indicate no (upper) or maximal (lower) inhibition from $A B$ to $L P$. The $w$-nullcline is the dotted curve. The trajectory is shown as a solid curve.

Additionally, the intersection of the $w$-nullcline with different $v$-nullclines forms a curve of fixed points, fp.c. B. The projection of the slow manifold (shaded) depicted in panel A onto the $w_{L P}-s_{A B}$ phase space. C. The projection of the $L P$ trajectory onto the $w_{L P}-s_{A B}-s_{P Y}$ phase space. The jump and fixed point surfaces, j.s. and fp.s., are now both two-dimensional. The trajectory begins toward the front of the box with a high value of $s_{P Y}$, but a low value of $s_{A B}$, and moves down, to the left, and into the box ( $s_{A B}, s_{P Y}$ and $w_{L P}$ all decay). When $A B$ becomes active, the trajectory is reset vertically to a higher value of $s_{A B}$. The trajectory then moves in the same way as before but for the time $A B$ is active, $s_{A B}$ decays very slowly. The trajectory escapes when it reaches j.s. (orange surface). 

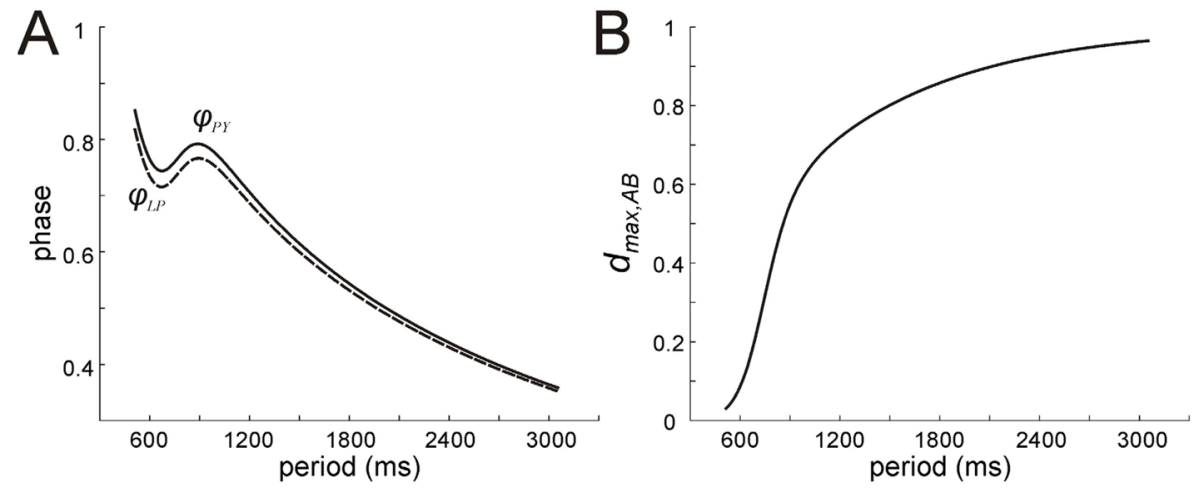

Fig. 5.

Behavior of $L P$ and $P Y$ when only $A B$ inhibition is present. A. Phase curves for $L P$ and $P Y$ show a stereotypical cubic shape indicating that different mechanisms and parameters control phase at different periods. B. The value of $d_{A B \text {, max }}$ increases with period. The strengthening of the feed-forward $A B$ synapses for $P>600$ causes the control of phase (as shown in panel A) to switch from the $L P$ and $P Y$ intrinsic mechanisms to the AB synapses. 


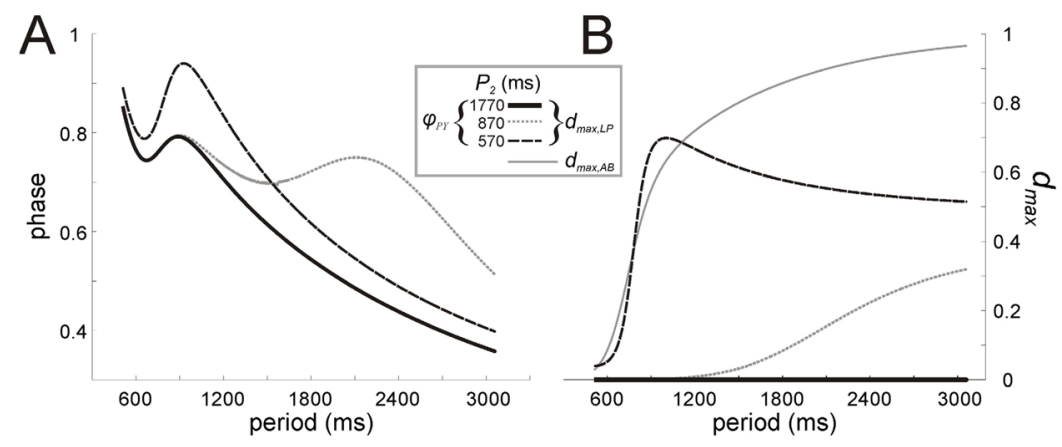

Fig. 6.

Behavior of $P Y$ under $A B$ and $L P$ inhibition. A. The $P Y$ phase curves shown in three conditions indicate how the half-activation period $\left(P_{2}\right)$ of the $L P$ to $P Y$ synapse affects its ability to change the phase of $P Y$. B. The behavior of $d_{L P, \max }$ for the three cases shown in panel A. The value $d_{A B, \max }$ (grey solid curve) is also shown to illustrate the relationship between the two synapses. 


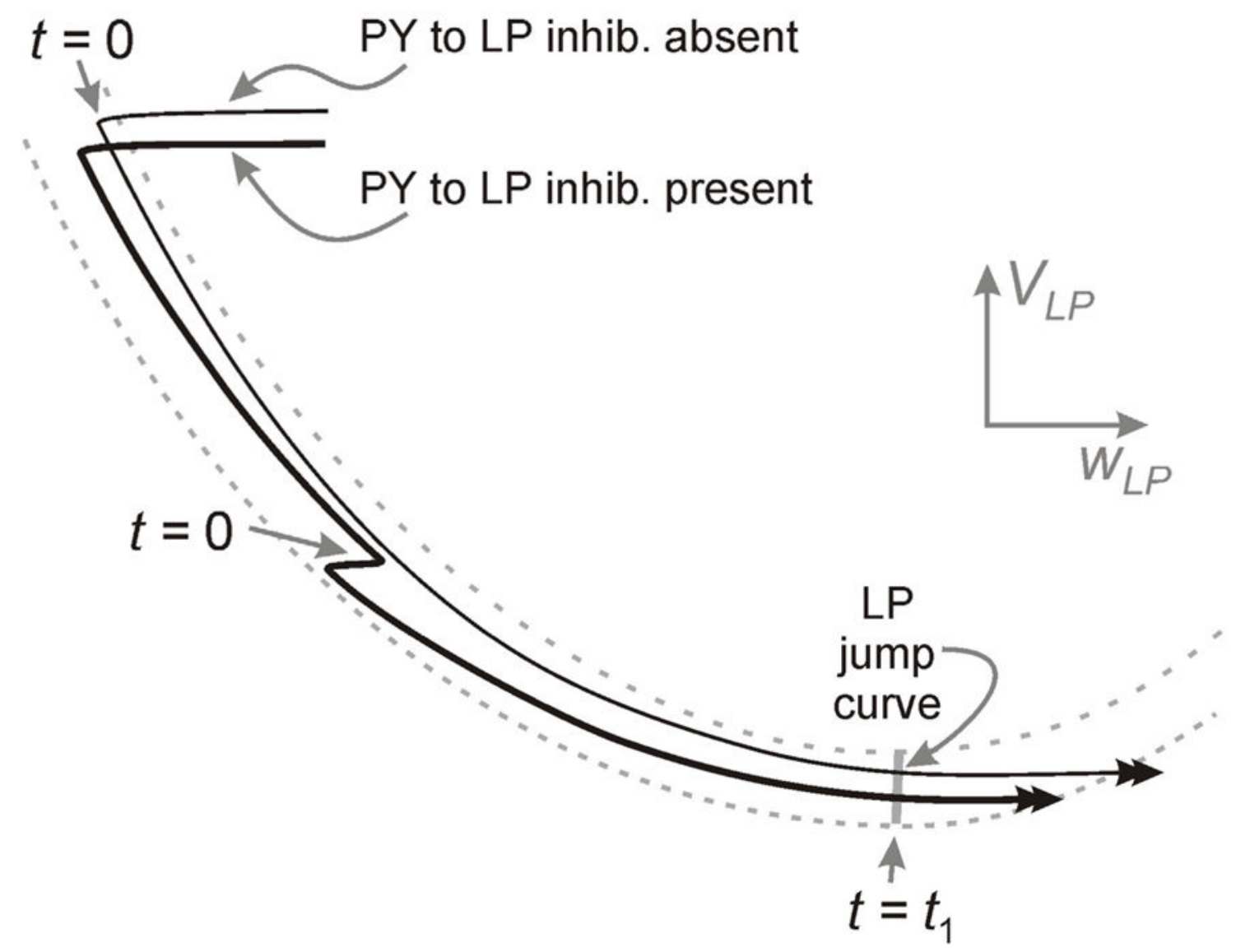

Fig. 7.

The behavior of $L P$ in the presence and absence of $P Y$ inhibition. Without $P Y$ inhibition, the time $t_{1}$ is calculated from the moment $L P$ falls back to the silent state, associated with the onset of $A B$ activity ( $\mathrm{t}=0$; top arrow) to the jump point. When $P Y$ inhibition is present (the bold trajectory), the onset of $A B$ activity occurs at a lower point on the left branch of the $v_{L P}$ nullcline $(\mathrm{t}=0$; middle arrow $)$ and $t_{1}$ is calculated from this moment to the jump point and is therefore much smaller. 

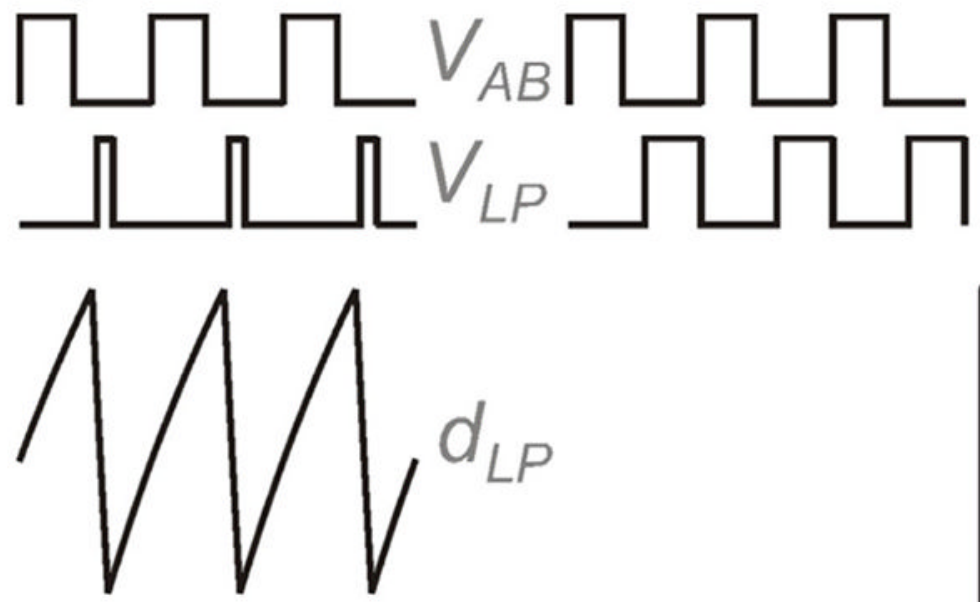

0.7

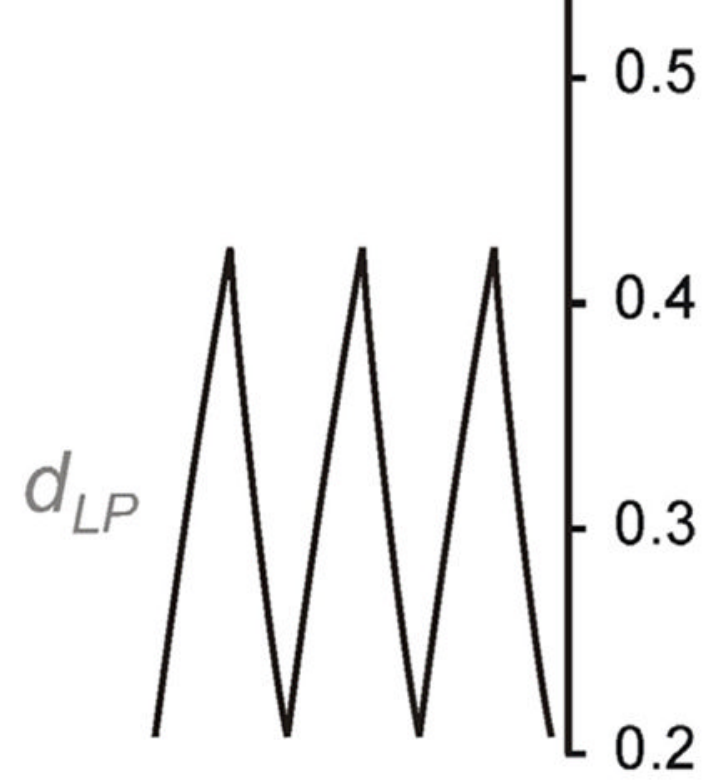

Fig. 8.

The effect of $P Y$ inhibition on $d_{L P}$. When $P Y$ inhibition is present, $L P$ inhibition strengthens independently of the half activation $\left(P_{2}\right)$ of the synapse since $L P$ spends more time in the silent state. When the $P Y$ inhibition is absent, the $L P$ inhibition can only strengthen if $P_{2}$ is sufficiently small as $L P$ spends relatively little time in the silent state. 

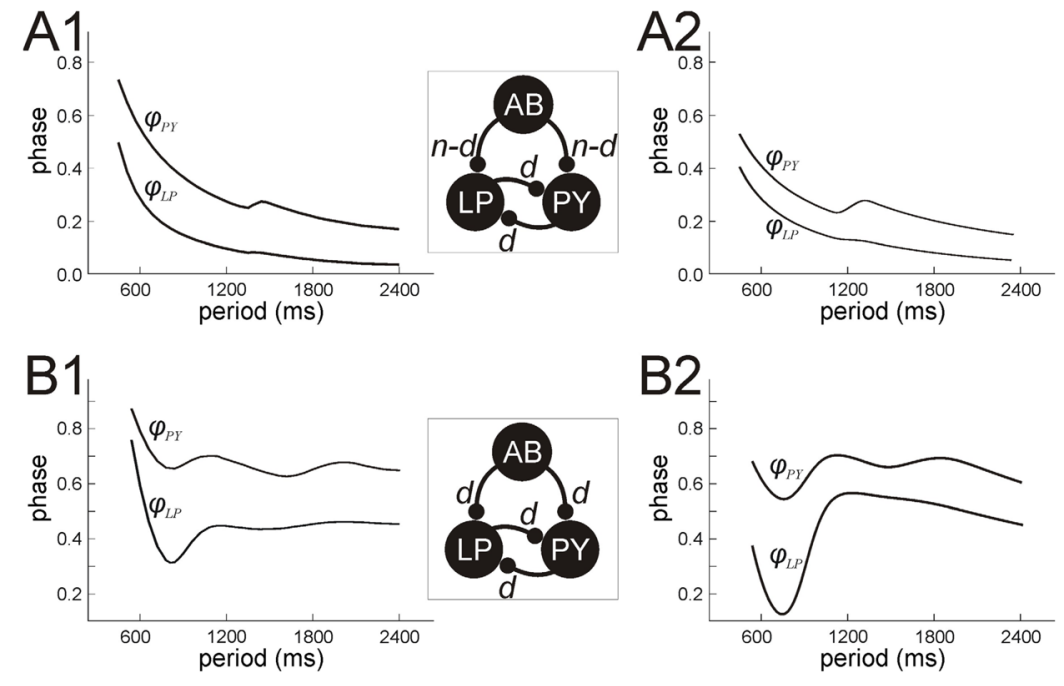

Fig. 9.

$L P$ and $P Y$ phase curves when reciprocal inhibition between the two is present. $\mathbf{A}$. When the $A B$ synapses are non-depressing, the $P Y$ phase can still be better than $1 / P$ if the $L P$ inhibition to $P Y$ is depressing. The local maxima of $\phi_{P Y}$ are due to the strengthening of the $L P$ to $P Y$ synapse. Also note the small increase in $\phi_{L P}$ near these periods. B. When the $A B$ synapses are depressing, $\phi_{P Y}$ and $\phi_{L P}$ can both have multiple local extrema. Panels A1 and B1 were obtained by numerically solving the full set of equations for their respective cases. Panels A2 and B2, obtained by solving equations (25) and (26), show good qualitative agreement with these numerical results. 